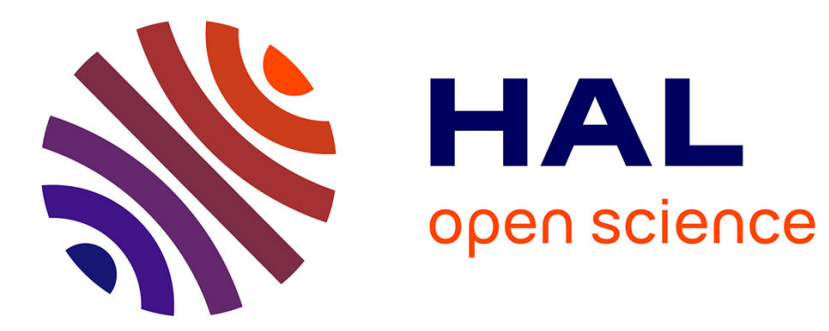

\title{
A Transposed-Word Effect in Same-Different Judgments to Sequences of Words
}

Felipe Pegado, Jonathan Grainger

\section{To cite this version:}

Felipe Pegado, Jonathan Grainger. A Transposed-Word Effect in Same-Different Judgments to Sequences of Words. Journal of Experimental Psychology: Learning, Memory, and Cognition, 2020. hal-02448237

\section{HAL Id: hal-02448237 \\ https://hal.science/hal-02448237}

Submitted on 24 Jan 2020

HAL is a multi-disciplinary open access archive for the deposit and dissemination of scientific research documents, whether they are published or not. The documents may come from teaching and research institutions in France or abroad, or from public or private research centers.
L'archive ouverte pluridisciplinaire HAL, est destinée au dépôt et à la diffusion de documents scientifiques de niveau recherche, publiés ou non, émanant des établissements d'enseignement et de recherche français ou étrangers, des laboratoires publics ou privés. 


\section{Journal of Experimental Psychology: Learning, Memory, and Cognition}

\section{A Transposed-Word Effect in Same-Different Judgments to Sequences of Words}

Felipe Pegado and Jonathan Grainger

Online First Publication, November 14, 2019. http://dx.doi.org/10.1037/xIm0000776

\section{CITATION}

Pegado, F., \& Grainger, J. (2019, November 14). A Transposed-Word Effect in Same-Different Judgments to Sequences of Words. Journal of Experimental Psychology: Learning, Memory, and Cognition. Advance online publication. http://dx.doi.org/10.1037/xIm0000776 



\title{
A Transposed-Word Effect in Same-Different Judgments to Sequences of Words
}

\author{
Felipe Pegado and Jonathan Grainger \\ CNRS and Aix-Marseille University
}

\begin{abstract}
The present study examined transposed-word effects in a same-different matching task with sequences of 5 words. The word sequences were presented one after the other, each for $400 \mathrm{~ms}$, the first in lowercase and the second in uppercase. The first sequence, the reference, was either a grammatically correct sentence or a scrambled ungrammatical sequence of the same words. The second sequence, the target, was either the same as the reference or differed either by transposing the second and third words or the third and fourth words in the first sequence or by replacing the same 2 words with different words in Experiment 1 or by a single word replacement in Experiment 2. The results showed that "same" responses were easier to make with grammatically correct references and that "different" responses were harder to make when the difference involved a transposition compared with a replacement. This transposed-word effect was found to be independent of reference grammaticality in Experiment 1. Experiment 2 again found a transposed-word effect for ungrammatical sequences, but here the effect was reduced compared with grammatical sequences. The effects found with ungrammatical sequences are taken to reflect the noisy bottom-up association of word identities to locations along a line of text, and this process combines with the influence of top-down grammatical constraints when "different" judgments are harder to make.
\end{abstract}

Keywords: transposed-words, same-different matching, multiword processing

To what extent can skilled readers process words in parallel when given the opportunity to do so? This question has been investigated with various paradigms in recent years (see Snell \& Grainger, 2019a, for a review of the evidence and a summary of the arguments in favor of using artificial paradigms to investigate basic processes in reading), and one finding in particular has been taken as evidence for parallel word processing in a relatively normal sentence reading situation. Mirault, Snell, and Grainger (2018) and Snell and Grainger (2019b) measured speeded grammaticality judgments to ungrammatical word sequences that were formed by transposing two words in a grammatically correct sentence (e.g., "The white cat was big" became "The white was cat big”). These grammaticality judgments were compared with judgments made to either an ungrammatical sequence that cannot be transformed into a correct sentence by a word transposition (e.g., "The white was cat slowly"; Mirault et al., 2018) or compared with ungrammatical sequences formed by transposing the two external words of the same sentence (e.g., "Big white cat was the"; Snell \& Grainger, 2019b). In both studies, it was found that the adjacent

Felipe Pegado and Jonathan Grainger, Laboratory of Cognitive Psychology, Institute for Language Communication and the Brain, CNRS and Aix-Marseille University.

This research was supported by ERC Grant 742141.

Correspondence concerning this article should be addressed to Felipe Pegado, Laboratory of Cognitive Psychology, Institute for Language Communication and the Brain, CNRS and Aix-Marseille University, 3 place Victor Hugo, 13331 Marseille, France. E-mail: felipe.pegado@univ-amu.fr transposed word sequences were harder to judge as being ungrammatical. These findings suggest that the adjacent transposed words provided evidence that these two words were in the grammatically correct order, hence making an ungrammatical decision harder (see Kennedy \& Pynte, 2008, for an earlier investigation of word order effects in reading). The present study was designed to investigate the different possible mechanisms at play in these transposed-word effects. It provides a further example of how artificial reading paradigms can help uncover processes that are not readily discernable in more natural reading conditions.

There are at least two mechanisms that could contribute to such word order effects during reading. One mechanism, postulated in the work of Snell, Meeter, and Grainger (2017), would involve the use of sentence-level structures to provide top-down syntactic constraints on the allocation of word identities to different spatiotopic locations along a line of text. These top-down constraints would contribute to implicitly reordering the transposed words, hence increasing the likelihood that participants perceive the word sequence as being grammatically correct. The other mechanism would involve adding noise to the bottom-up process that allocates word identities to different spatiotopic locations (Mirault et al., 2018). That is, in an account of sentence reading where word identities are assigned to different spatiotopic locations along a line of text (Snell et al., 2017; Snell, van Leipsig, Grainger, \& Meeter, 2018), this assignment process would be subject to a certain amount of noise such that a given word identity would be associated not only with its true location but also with neighboring positions, albeit with a lower likelihood (see Gomez, Ratcliff, \& Perea, 2008, for an analogous account of noisy letter position coding). 
The first demonstrations of transposed-word effects, described above, used a speeded grammaticality judgment task. This task explicitly requires the computation of syntactic information and therefore might have exaggerated the role that such structures played in generating the observed transposed-word effect. In an attempt to disentangle the relative contribution of top-down sentence-level constraints and noisy bottom-up allocation of word identities to word locations, in the present study, we used a task that does not require the computation of syntactic structures - the same-different matching task. This task involves the brief presentation of a first stimulus, the reference, followed by the brief presentation of a second stimulus, the target, and participants must judge if the reference and target are the same or not. Using the same-different matching task allowed us to compare transposedword effects obtained in conditions where sentence-level constraints can operate (i.e., using a grammatically correct reference) with conditions where they cannot (i.e., using an ungrammatical reference).

In sum, the present study was designed to separate out the relative contribution of two factors that are hypothesized to be driving the transposed-word effects observed in prior work using speeded grammaticality judgments: (a) the noisy-bottom up association of word identities to word locations and (b) the top-down use of sentence-level structures to constrain the association of word identities to plausible locations. Experiment 1 compared the effects of transposing two words with a condition where the same two words were replaced by different words. Experiment 2 compared the effects of transposing two words with a condition where only one of those words was replaced by a different word. Transposed-word effects found with an ungrammatical reference would be evidence for a role for bottom-up processes, and a greater transposed-word effect obtained with grammatical compared with ungrammatical references would be evidence for top-down processes.

\section{Experiment 1}

\section{Method}

Participants. Twenty-eight participants (21 females) were recruited at Aix-Marseille University (Marseille, France). All participants were native speakers of French. They received monetary compensation (10 €/hour) or course credit. All reported normal or corrected-to-normal vision, ranged in age from 18 to 33 years $(M=21.96$ years, $S D=3.04)$, and signed informed-consent forms prior to participation. Ethics approval was obtained from the "Comité de Protection des Personnes SUD-EST IV" (No. 17/051).

Design and stimuli. We created 40 different sentences in French. Each of these sentences was composed of five words. The 40 sentences were used to generate an equivalent number of ungrammatical sequences by scrambling the order of words. These 40 sentences and 40 ungrammatical sequences formed the set of sequences that were presented as the first of two sequences on each trial, called the reference. For every reference, we generated three types of target sequence (the second sequence on each trial), for a total of 240 trials. The three types of target were (a) repetition-the same sequence as the reference; (b) transposition-the words at Positions 2 and 3 or Positions 3 and 4 in the reference were flipped; and (c) replacement - the words at Positions 2 and 3 or Positions 3 and 4 in the reference were replaced with different words. The replacement words had the same length, syntactic function, and word frequency (on average) as the words they replaced. The average length of these critical words was 4.54 letters (range: 1-6 letters), and the average frequency based on values obtained from Lexique2 (New, Pallier, Brysbaert, \& Ferrand, 2004) was 6.50 on the Zipf scale of van Heuven, Mandera, Keuleers, and Brysbaert (2014), with a range of 2.85-7.51 Zipf. The design involved distinct analyses for the "same" response trials and the "different" response trials. The "same" response analysis contrasted grammatical and ungrammatical references (Reference Grammaticality factor). The "different" response analysis involved a 2 (Reference Grammaticality) $\times 2$ (Type of Change) design. Table 1 provides examples of reference and target sequences used in the "different" response conditions in the experiment (French) and also in English for expository purposes. For each participant, every reference was repeated three times associated with one of its three target sequences (one same response, two types of different response). With 80 trials per condition and 28 participants, the number of data points per condition exceeded that recommended by Brysbaert and Stevens (2018).

Apparatus. Stimuli were presented using OpenSesame (Version 3.0.7; Mathôt, Schreij, \& Theeuwes, 2012) and displayed on a 47.5 -cm $\times 27-\mathrm{cm} \mathrm{LCD} \mathrm{screen}(1,024 \times 768$ pixels resolution $)$. Participants were seated about $70 \mathrm{~cm}$ from the monitor, such that every four characters (monospaced font in black on a gray background) equaled approximately $1^{\circ}$ of visual angle. Responses were

Table 1

Examples of the Reference and Target Sequences for the "Different" Response Trials in Experiment 1

\begin{tabular}{lll}
\hline \multicolumn{1}{c}{ Reference } & Examples from the experiment & Examples to illustrate the design \\
\hline Grammatical reference & il veut ces pommes vertes & he wants these green apples \\
Transposed word target & IL CES VEUT POMMES VERTES & HE THESE WANTS GREEN APPLES \\
Replaced word target & IL DIRA MES POMMES VERTES & HE TALKS THEIR GREEN APPLES \\
Ungrammatical reference & vertes veut ces il pommes & green wants these he apples \\
Transposed word target & VERTES CES VEUT IL POMMES & GREEN THESE WANTS HE APPLES \\
Replaced word target & VERTES DIRA MES IL POMMES & GREEN TALKS THEIR HE APPLES \\
\hline
\end{tabular}

Note. Not shown here is the condition where targets were the same word sequence as the reference but printed in uppercase (i.e., "same" response trials). The transpositions and replacements operate on the second and third words in these examples and could equally be on the third and fourth words in the experiment. 
recorded via a computer keyboard (keys "j" for the right and "f" for the left index fingers).

Procedure. The experiment took place in a quiet room. The instructions were given both by the experimenter and on screen. On every trial, participants had to decide if the two sequences presented one after the other on the computer screen were the same or different, where "same" was defined as being composed of the same words in the same order (see Figure 1). A training phase was performed before the experiment to ensure good comprehension and familiarization with the task. The first sequence, the reference, was always presented in lowercase, while the second sequence, the target, was always shown in uppercase, in order to avoid purely visual matching. In order to compensate for the difference in the size of lowercase and uppercase letters, the font size of the reference was slightly greater than that of the target (24 pixels and 22 pixels, respectively) such that one character corresponded to approximately $0.3 \mathrm{~cm}$ in both cases. All stimuli were presented in droid monospaced font, the default font for OpenSesame. The words in each sequence were presented simultaneously for a duration of $400 \mathrm{~ms}$. Targets immediately followed the reference. The position of the reference was slightly higher than the central fixation cross, and the position of the target sequence was slightly lower, such that the two sequences were separated by approximately one line of text. Participants were requested to respond as fast and as accurately as possible. Each trial started with a fixation cross for $300 \mathrm{~ms}$ followed by the reference for $400 \mathrm{~ms}$, followed by the target for $400 \mathrm{~ms}$, followed by a question mark "?" presented until the participants' answer (or for a maximum of $5 \mathrm{~s}$ ). Then a neutral gray screen was displayed for $200 \mathrm{~ms}$ and a new trial started.

\section{Results}

Participants presented an overall error rate of $23.0 \%$ and a median response time (RT) from target onset restricted to correct responses of $923 \mathrm{~ms}$ (trials with RTs longer than 3,000 ms after target offset were excluded). Statistical analysis of error rates and $\log _{10}$-transformed RTs were performed using R software (Version 3.5.1), separately for "same" and "different" responses. Linear mixed effects (LME) model analysis with random effects for subjects and items was performed with the lme4 library (Bates, Mächler, Bolker, \& Walker, 2015), trying to maximize randomness by including whenever possible random slopes in addition to random intercepts (Barr, Levy, Scheepers, \& Tily, 2013). We report $b$ values, standard errors, and $t$ values (for RTs) and $z$ values (for error rates), with $t$ and $z$ values beyond |1.96| deemed significant (Baayen, 2008). A complementary Bayes factor analysis was performed using the BayesFactor library. Condition means are shown in Figure 2.

"Same" response trials. For trials requiring a "same" response, we performed LME model analysis by declaring Reference Grammaticality (grammatical vs. ungrammatical sequences) as a fixed-effect factor. The models were fully randomized for errors and RTs. We found a significant effect of Grammaticality for errors $(b=0.99, S E=0.21, z=4.66)$ and RTs $(b=0.05, S E=$ $0.01, t=4.32$ ), indicating that ungrammatical sequences were more difficult to judge than grammatically correct sentences (respectively $17.5 \%$ vs. $8.11 \%$ errors and 1,113 vs. $1,001 \mathrm{~ms}$ ).

"Different" response trials. We then moved for the critical part of the data: that is, for trials requiring a "different" response. LME model analysis was performed by declaring Type of Change (transposition vs. replacement) and Reference Grammaticality as fixed-effect factors. For errors, the model had random intercepts for all factors but random slopes only by participant for Type of Change because more complex models did not converge. We found significant main effects of Reference Grammaticality $(b=0.69, S E=0.21, z=3.32)$ and Type of Change ( $b=3.21, S E=0.20, z=15.7)$. Participants made more errors with grammatically incorrect references $(30.6 \%)$ than grammatically correct references $(25.7 \%)$. They made 10 -

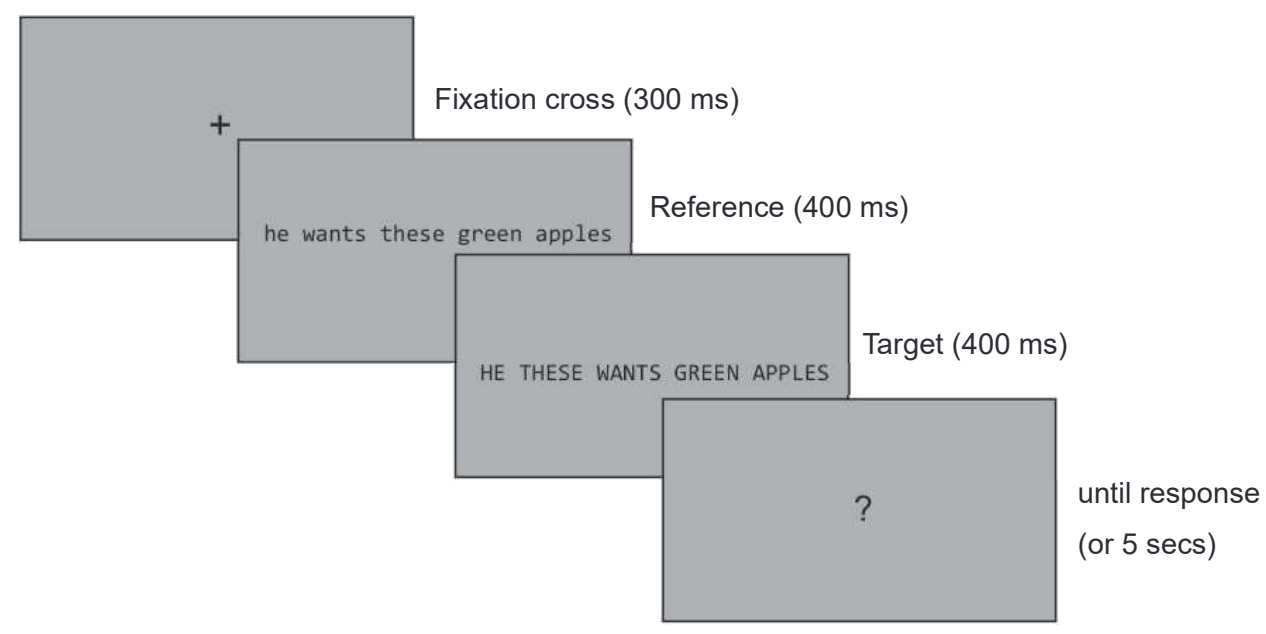

Figure 1. The same-different matching paradigm applied to sequences of words. Participants had to decide as rapidly and as accurately as possible if the two sequences of words were the "same" or "different" by pressing appropriate response keys. In this example, the target sequence differed from the reference sequence by the transposition of the second and third words. 
Errors ["same"]

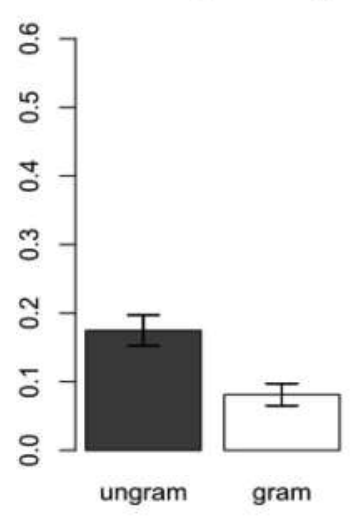

RTs ["same"]



\section{Errors ["different"]}

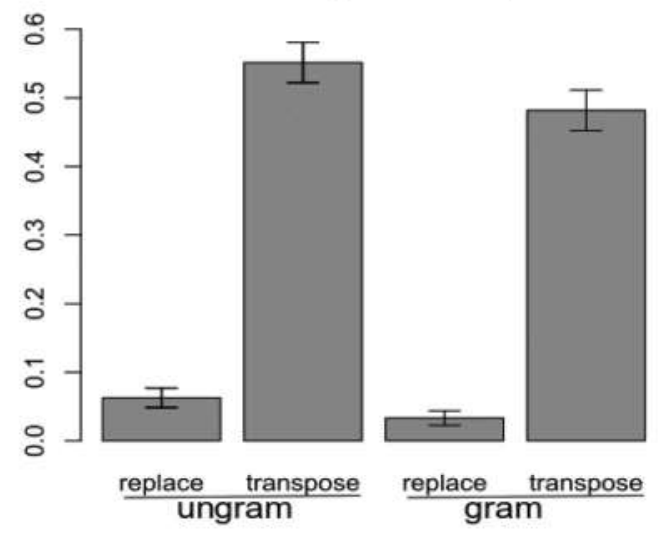

RTs ["different"]

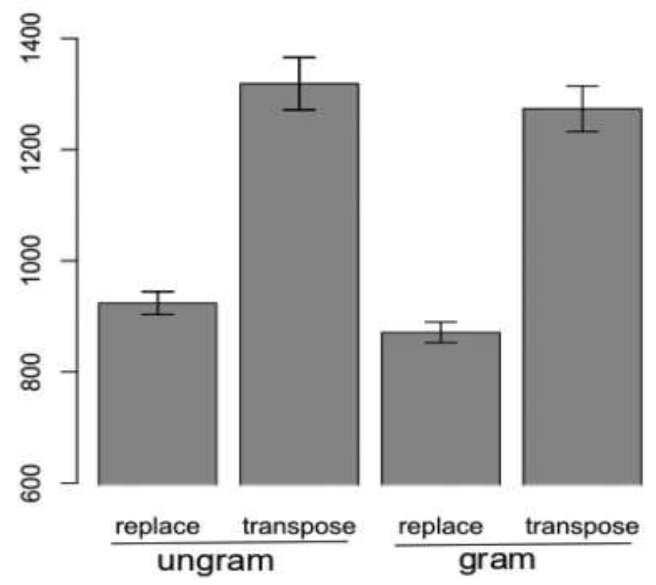

Figure 2. Results of Experiment 1. Error rates in probabilities (upper panel) and response times (RTs) in milliseconds (lower panel), for "same" response trials (left) and "different" response trials (right) as a function of reference grammaticality (ungrammatical vs. grammatical) and type of change (replace vs. transpose). Error bars represent $95 \%$ confidence intervals.

fold more errors when the change involved a transposition $(51.7 \%)$ compared with a replacement $(4.80 \%)$. The interaction between these two factors was not significant $(b=0.36, S E=$ $0.23, z=1.57$ ). To confirm this null interaction, we performed a Bayes factor analysis, using Bayesian information criteria (Wagenmakers, 2007), which compares the fit of the data under the null hypothesis relative to the alternative hypothesis. Results revealed that the data were 4.57 times more likely to occur under the null than the alternative hypothesis for the interaction term, providing positive evidence for the lack of interaction.

LME analysis of RTs (with random intercepts only) showed again main effects of Type of Change $(b=0.16, S E=0.007, t=$ $24.5)$, and Reference Grammaticality ( $b=0.02, S E=0.005, t=$ 4.37) and no interaction $(b=0.01, S E=0.009, t=1.22)$. Participants took longer to respond correctly on transposed-word trials $(1,294 \mathrm{~ms})$ than replacement trials $(897 \mathrm{~ms})$. They also took longer to respond with ungrammatical references $(1,051 \mathrm{~ms})$ than grammatical references $(1,011 \mathrm{~ms})$. We again tested the lack of interaction with a Bayes factor analysis, and this revealed that the data were 11.7 times more likely to occur under the null than the alternative hypothesis for the interaction term.

\section{Discussion}

Experiment 1 revealed robust transposed-word effects in a same-different matching task in which target sequences of words were compared with reference sequences that could either be grammatically correct or not. Although reference grammaticality affected both "same" and "different" decisions, with faster and more accurate responses with grammatically correct references, it did not impact on the size of transposed-words effect seen in "different" responses. The difference between a reference and its transposed-word target was equally hard to discern when the reference was an ungrammatical sequence of words compared with correct sentences. This points to a key role for bottom-up processes in generating transposed-word effects.

The results of Experiment 1 failed to support the prediction that transposed-word effects would be greater when the reference is a grammatically correct sequence. However, it is possible that the two-word replacement condition used in Experiment 1 was too easy to detect, hence encouraging a purely bottom-up strategy for generating different responses in this experiment. In Experiment 2, we made the replacement condi- 
tion harder by combining a transposition manipulation with a replacement manipulation such that replacement targets differed from transposition targets by only one word that was from the same syntactic category.

\section{Experiment 2}

\section{Method}

Participants. Twenty-eight participants ( 22 females) were recruited at Aix-Marseille University (Marseille, France). All participants were native speakers of French. They received monetary compensation (10 €/hour). All reported normal or corrected-tonormal vision, ranged in age from 18 to 30 years $(M=22.9$ years, $S D=2.39$ ), and signed informed-consent forms prior to participation. Ethics approval was obtained from the "Comité de Protection des Personnes SUD-EST IV" (No. 17/051).

Design and stimuli. The same 40 grammatical and ungrammatical reference sequences used in Experiment 1 were again used here, leading to the same total of 240 trials. The same three types of reference-target relation were manipulated: repetition, transposition, and replacement. The only difference with Experiment 1 concerned the replacement condition, where we now replaced just one word instead of two, and to be comparable with the transposition condition, we also transposed one word in an equivalent way (see Table 2).

Apparatus and procedure. These were the same as in Experiment 1 .

\section{Results}

Participants presented an overall error rate of $35.2 \%$ and a median RT from target onset restricted to correct responses of 928 ms (trials with RTs longer than 3,000 ms after target offset were excluded). Statistical analysis of error rates and $\log _{10}$-transformed RTs were performed using R software (Version 3.5.1), separately for "same" and "different" responses using LME models as in Experiment 1 . We report $b$ values, standard errors, and $t$ values (for RTs) and $z$ values (for error rates), with $t$ and $z$ values beyond $|1.96|$ deemed significant. Condition means are shown in Figure 3.

"Same" response trials. For trials requiring a "same" response, we performed a LME model analysis by declaring Reference Grammaticality (grammatical vs. ungrammatical sequences) as the fixed-effect factor and participants and items as random factors (with random intercepts and slopes for errors, as well as by-participant random intercepts and slopes and by-item random intercepts only for RTs as the fully randomized model did not converge). We found a significant effect of Reference Grammaticality both for errors ( $b=1.10, S E=0.20, z=5.59)$ and RTs ( $b=0.04, S E=0.008, t=4.79)$, indicating that "same" decisions were harder to make with ungrammatical references than grammatically correct references (respectively $26.3 \%$ vs. $12.8 \%$ errors and 1,107 vs. $1,062 \mathrm{~ms}$ ).

"Different" response trials. For trials requiring a "different" response, LME analyses were performed by declaring Type of Change (transposition vs. replacement) and Reference Grammaticality as fixed-effect factors and participants and items as random factors. For errors, random slopes could not be included for the interaction term because of convergence issues. For RTs, the model included only random intercepts because more complex models did not converge.

For errors, we found a main effect of Type of Change ( $b=0.90$, $S E=0.22, z=4.14)$, with greater error rates when the change involved a transposition (54.0\%) compared with a replacement $(31.7 \%)$. The main effect of Reference Grammaticality failed to reach significance $(b=0.29, S E=0.15, z=1.91 ; 43.3 \%$ for grammatical and $42.3 \%$ for ungrammatical sequences), but there was a significant interaction $(b=0.58, S E=0.14, z=4.15)$. Transposition effects, that is, the difference between the transposed and replaced conditions, were greater with grammatically correct references $(27.7 \%)$ compared with ungrammatical references $(21.3 \%)$.

The RT analyses showed main effects of Type of Change ( $b=$ $0.04, S E=0.008, t=5.18)$ and Reference Grammaticality $(b=$ $0.02, S E=0.007, t=2.63)$ that were also qualified by a significant interaction $(b=0.04, S E=0.01, t=3.23)$. Participants took longer to respond correctly on transposed-word trials $(1,183 \mathrm{~ms})$ than replacement trials $(1,019 \mathrm{~ms})$. They also took longer for ungrammatical $(1,107 \mathrm{~ms})$ than grammatical $(1,062 \mathrm{~ms})$ references. As with the error analyses, transposition effects were greater with grammatically correct references ( $227 \mathrm{~ms})$ compared with ungrammatical references $(101 \mathrm{~ms})$.

\section{Discussion}

The results of Experiment 2 are clear-cut. We replicated the finding of Experiment 1 that "same" decisions are easier to make for grammatical sequences compared with ungrammatical se-

Table 2

Examples of the Reference and Target Sequences for the "Different" Response Trials in Experiment 2

\begin{tabular}{|c|c|c|}
\hline Reference & Examples from the experiment & Examples to illustrate the design \\
\hline Grammatical reference & votre jeune tante danse bien & your young aunt dances well \\
\hline Transposed word target & VOTRE TANTE JEUNE DANSE BIEN & YOUR AUNT YOUNG DANCES WELL \\
\hline Replaced word target & VOTRE TANTE VIEUX DANSE BIEN & YOUR AUNT OLDER DANCES WELL \\
\hline Ungrammatical reference & danse jeune tante bien votre & dances young aunt well your \\
\hline Transposed word target & DANSE TANTE JEUNE BIEN VOTRE & DANCES AUNT YOUNG WELL YOUR \\
\hline Replaced word target & DANSE TANTE VIEUX BIEN VOTRE & DANCES AUNT OLDER WELL YOUR \\
\hline
\end{tabular}


Errors ["same"]

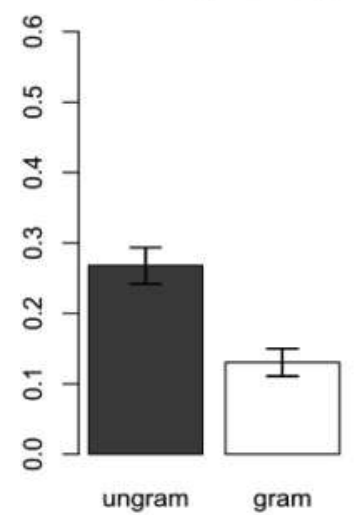

RTs ["same"]



Errors ["different"]

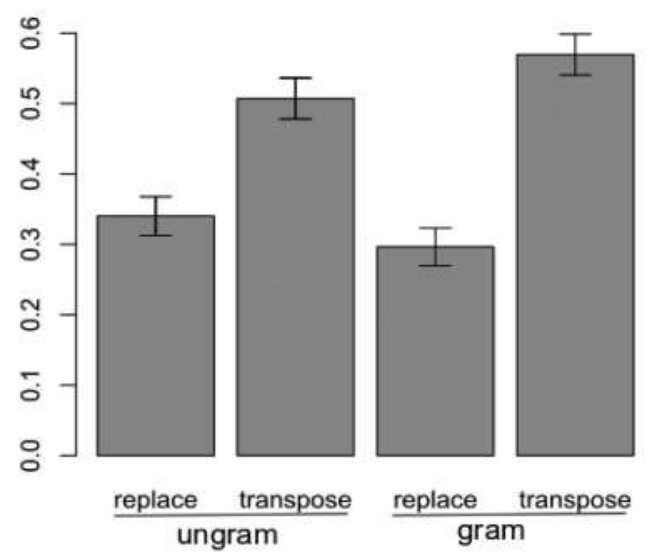

RTs ["different"]



Figure 3. Results of Experiment 2. Error rates in probabilities (upper panel) and response times (RTs) in milliseconds (lower panel), for "same" response trials (left) and "different" response trials (right) as a function of reference grammaticality (ungrammatical vs. grammatical) and type of change (replace vs. transpose). Error bars represent $95 \%$ confidence intervals.

quences. We further replicated the finding that ungrammatical decisions are harder to make when the ungrammaticality is caused by transposing two words compared with the word replacement condition - a transposed-word effect. Crucially, however, by making the replacement condition harder in Experiment 2, we now obtain a significant interaction between reference grammaticality and transposed-word effects. Transposed-word effects were significantly greater when the reference was a correct sentence compared with ungrammatical references. This provides clear evidence for a role for top-down syntactic influences on same-different judgments to word sequences, with grammatically correct references imposing syntactic constraints that modify participants' perception of the similarity of the reference and target sequences.

\section{General Discussion}

The present study examined the effects of word transpositions in a same-different matching task in which a first sequence of five words (the reference sequence) was briefly presented and immediately followed by a second sequence of five words (the target sequence) that could either be the same as the first or a different sequence. The first sequence of words could either be a grammatically correct sentence or an ungrammatical scrambled version of the same words. The second sequence could differ from the first either by a transposition of the second and third or third and fourth words in the sequence, or by replacing the same two words with different words in Experiment 1 or replacing only one of the transposed words in Experiment 2. In both experiments, "same" responses were easier to make with grammatically correct sentences compared with ungrammatical sequences. Crucially, when examining the different judgments, we found that these were harder to make when the difference involved a word transposition compared with a word replacement. This constitutes the first demonstration of a transposed-word effect in a same-different judgment task - a task that does not require the computation of syntactic structures.

On the basis of prior work on transposed-word effects in a grammaticality judgment task (Mirault et al., 2018; Snell \& Grainger, 2019b), and within the theoretical framework proposed 
by Snell et al. (2017), we predicted a role for two mechanisms in driving such effects: (a) the noisy bottom-up association of word identities to spatiotopic locations along a line of text and (b) syntactic structures imposing top-down constraints on word order. The present study tested the contribution of bottom-up processes by examining transposed-word effects with ungrammatical sequences of words. The results are unequivocal. Robust transposedword effects were found with ungrammatical references in both experiments. As concerns the role of top-down syntactic constraints, Experiment 1 failed to find evidence for the critical interaction between transposed-word effects and reference grammaticality. Transposition effects were statistically equivalent when the reference was a correct sentence and when it was an ungrammatical scrambled sequence of the same words (see Table 1 for examples and Figure 3 for the results). This suggests that top-down sentence-level constraints were not contributing to the transposition effects in that experiment. The first conclusion of the present work is therefore that one of the mechanisms driving transposedword effects in the same-different judgment task is the noisy bottom-up association of word identities to different locations along a line of text and that this mechanism is most likely a key component of transposed-word effects independently of the paradigm used to reveal such effects.

However, the failure to find evidence for top-down influences in Experiment 1 might have been due to the double replacement condition being too easy to detect and leading to participants opting for a purely bottom-up strategy for making "different" decisions. In order to test this possibility, in Experiment 2, we increased the difficulty of the replacement condition by replacing only one of the two transposed words in the transposed condition with a different word (see Table 2 for examples and Figure 3 for the results). Transposition effects were numerically reduced in Experiment 2 but were robust. Crucially, we now observed an interaction between reference grammaticality and the size of transposition effects, with the effects being greater when the reference sequence was a grammatically correct sentence. This suggests that reference grammaticality induced a bias to reorder the transposed words in the target sequence such as to form a correct sentence. This would provide evidence that the target formed the same word sequence as the reference, hence making ungrammatical decisions harder. Furthermore, both the interaction between reference grammaticality and transposition effects seen in "different" responses in Experiment 2 and the main effect of grammaticality on "same" decisions seen in both experiments clearly demonstrate that participants were generating sentence-level representations despite the fact that these were not necessary for the task. This points to an irrepressible process of syntactic computation that is automatically initiated when a sequence of words is present.

Just like transposed-letter effects point to parallel processing of letter stimuli (see Grainger, 2008, for a summary of the evidence), we argue that transposed-word effects plead in favor of parallel processing of words. It is the parallel processing of word identities combined with the noisy allocation of these identities to different spatiotopic locations and the rapid computation of syntactic structures that is thought to be driving the transposed-word effects seen in the present experiments. If words were identified sequentially, then their sequential identification should provide error-free information about word order, and no transposed-word effects should be observed (Reichle, Liversedge, Pollatsek, \& Rayner, 2009;
Snell \& Grainger, 2019a). Of course, the same-different judgment task used in the present work differs radically from more natural sentence reading. We used this task in order to explore the mechanisms driving transposed-word effects that are observed in an arguably more natural reading context (Mirault et al., 2018; Snell \& Grainger, 2019b). ${ }^{1}$ One possibility, however, is that participants in the Mirault et al. (2018) study were reading the transposed-word sequences sequentially but out of order, skipping the first word in order to fixate the second word before regressing back to the first word. This noncanonical order of word fixations would then generate the illusion of grammaticality. However, this hypothesis has been tested and rejected in a recent eye-movement study using the same materials and task as the Mirault et al. (2018) study (Mirault, Guerre-Genton, Dufau, \& Grainger, 2019). Although there was a significant proportion of out-of-order fixations, in line with the corpus analysis of Kennedy and Pynte (2008), the transposed-word effect remained robust when the out-of-order trials were removed prior to analysis. Nevertheless, we acknowledge that a serial model of reading might be able to account for transposed-word effects by adding noise to the process that transfers word-order information to higher-level syntactic processes on the basis of the order in which words are identified.

Given the role for bottom-up processes in driving transposedword effects in same-different matching, we are led to predict that similar transposition effects should be obtained with other kinds of stimuli such as sequences of pictures of familiar objects. This is in line with general accounts of location uncertainty in visual perception, such as proposed by Ratcliff (1981; see also Ashby, Prinzmetal, Ivry, \& Maddox, 1996; Compton \& Logan, 1993). Indeed, Ratcliff's (1981) theory was developed on the basis of results obtained with same-different judgments to strings of letters, and as noted earlier, the subsequent development of that theory to account for transposed-letter effects (Gomez et al., 2008) is analogous to the bottom-up mechanism we propose to account for transposed-word effects. In line with a general principle of object location uncertainty is the fact that transposition effects in samedifferent judgments have been found with other kinds of visual objects such as digits and symbols (Duñabeitia, Dimitropoulou, Grainger, Hernández, \& Carreiras, 2012; Massol, Duñabeitia, Carreiras, \& Grainger, 2013) and musical notes in the staff (Perea, García-Chamorro, Centelles, \& Jiménez, 2013). However, there is also evidence for reading-specific mechanisms for location encoding. For example, transposition effects in same-different matching are greater with random consonant strings than with either digit or symbol strings (see Grainger, 2018, for a summary of the evidence), and letter stimuli are particularly sensitive to string orientation (Ktori, Bertrand, \& Grainger, 2019). We therefore predict that word stimuli should also show a reading-specific pattern when comparing random word sequences with sequences of pictures of objects, for example, and that transposed-word effects should reveal a greater sensitivity to the specific axis along which the words are aligned (e.g., horizontal vs. vertical). Furthermore, given the constraints operating on location uncertainty, we also predict that transpositions of nonadjacent words in the same-different

\footnotetext{
${ }^{1}$ We note nevertheless that Rayner, Angele, Schotter, and Bicknell (2013) failed to find strong evidence for transposed-word effects in a sentence reading paradigm with a parafoveal preview manipulation.
} 
judgment task should lead to a reduced effect, as has been observed in speeded grammaticality judgments (Snell \& Grainger, 2019b).

In future work examining the interplay between bottom-up and top-down influences on transposed-word effects, it will be interesting to study cases where the transposition generates a grammatically correct sequence, such as when transposing the two adjectives in "The thin tall man stood up," which becomes "The tall thin man stood up." Here, we predict an increase in the size of transposedword effects, because the transposed words are syntactically possible words at both locations. Also, future studies using high temporal resolution neuroimaging techniques such as electroencephalography could examine the temporal dynamics of transposed-word effects seen in the same-different matching task. This could further elucidate the relative contribution of early bottom-up processes relative to later top-down processes.

In conclusion, the present work demonstrates how the samedifferent judgment task can be usefully applied to investigate word-order encoding during sentence reading, similarly to the way that this task has been applied in prior research to investigate letter-order encoding. Parallel to the letter-order results, we found evidence for the noisy association of word identities to spatiotopic locations in a sequence of words, such that evidence for a word at a given location could also be taken as evidence for that word at a neighboring location. We also found evidence for a role for topdown syntactic constraints on word order. We conclude that transposed-word effects reflect the combined operation of these two mechanisms.

\section{References}

Ashby, F. G., Prinzmetal, W., Ivry, R., \& Maddox, W. T. (1996). A formal theory of feature binding in object perception. Psychological Review, 103, 165-192. http://dx.doi.org/10.1037/0033-295X.103.1.165

Baayen, R. (2008). Analyzing linguistic data: A practical introduction to statistics. New York, NY: Cambridge University Press. http://dx.doi .org/10.1017/CBO9780511801686

Barr, D. J., Levy, R., Scheepers, C., \& Tily, H. J. (2013). Random effects structure for confirmatory hypothesis testing: Keep it maximal. Journal of Memory and Language, 68, 255-278. http://dx.doi.org/10.1016/j.jml .2012.11.001

Bates, D., Mächler, M., Bolker, B., \& Walker, S. (2015). Fitting linear mixed-effects models using lme4. Journal of Statistical Software, 67, 1-48. http://dx.doi.org/10.18637/jss.v067.i01

Brysbaert, M., \& Stevens, M. (2018). Power analysis and effect size in mixed effects models: A tutorial. Journal of Cognition, 1, 1-20.

Compton, B. J., \& Logan, G. D. (1993). Evaluating a computational model of perceptual grouping by proximity. Perception \& Psychophysics, 53, 403-421. http://dx.doi.org/10.3758/BF03206783

Duñabeitia, J. A., Dimitropoulou, M., Grainger, J., Hernández, J. A., \& Carreiras, M. (2012). Differential sensitivity of letters, numbers, and symbols to character transpositions. Journal of Cognitive Neuroscience, 24, 1610-1624. http://dx.doi.org/10.1162/jocn_a_00180

Gomez, P., Ratcliff, R., \& Perea, M. (2008). The overlap model: A model of letter position coding. Psychological Review, 115, 577-600. http:// dx.doi.org/10.1037/a0012667

Grainger, J. (2008). Cracking the orthographic code: An introduction. Language and Cognitive Processes, 23, 1-35. http://dx.doi.org/10.1080/ 01690960701578013
Grainger, J. (2018). Orthographic processing: A 'mid-level' vision of reading: The 44th Sir Frederic Bartlett Lecture. Quarterly Journal of Experimental Psychology, 71, 335-359. http://dx.doi.org/10.1080/17470218.2017 .1314515

Kennedy, A., \& Pynte, J. (2008). The consequences of violations to reading order: An eye movement analysis. Vision Research, 48, 2309-2320. http://dx.doi.org/10.1016/j.visres.2008.07.007

Ktori, M., Bertrand, D., \& Grainger, J. (2019). What's special about orthographic processing? Further evidence from transposition effects in same-different matching. Quarterly Journal of Experimental Psychology, 72, 1780-1789. http://dx.doi.org/10.1177/1747021818811448

Massol, S., Duñabeitia, J. A., Carreiras, M., \& Grainger, J. (2013). Evidence for letter-specific position coding mechanisms. PLOS ONE, 8, e68460. http://dx.doi.org/10.1371/journal.pone.0068460

Mathôt, S., Schreij, D., \& Theeuwes, J. (2012). OpenSesame: An opensource, graphical experiment builder for the social sciences. Behavior Research Methods, 44, 314-324. http://dx.doi.org/10.3758/s13428-0110168-7

Mirault, J., Guerre-Genton, A., Dufau, S., \& Grainger, J. (2019). Using virtual reality to study reading: An eye-tracking investigation of transposed-word effects. Manuscript submitted for publication.

Mirault, J., Snell, J., \& Grainger, J. (2018). You that read wrong again! A transposed-word effect in grammaticality judgments. Psychological Science, 29, 1922. http://dx.doi.org/10.1177/0956797618806296

New, B., Pallier, C., Brysbaert, M., \& Ferrand, L. (2004). Lexique 2: A new French lexical database. Behavior Research Methods, Instruments \& Computers, 36, 516-524. http://dx.doi.org/10.3758/BF03195598

Perea, M., García-Chamorro, C., Centelles, A., \& Jiménez, M. (2013). Position coding effects in a 2D scenario: The case of musical notation. Acta Psychologica, 143, 292-297. http://dx.doi.org/10.1016/j.actpsy.2013.04.014

Ratcliff, R. (1981). A theory of order relations in perceptual matching. Psychological Review, 88, 552-572. http://dx.doi.org/10.1037/0033-295X.88.6 .552

Rayner, K., Angele, B., Schotter, E. R., \& Bicknell, K. (2013). On the processing of canonical word order during eye fixations in reading: Do readers process transposed word previews? Visual Cognition, 21, 353381. http://dx.doi.org/10.1080/13506285.2013.791739

Reichle, E. D., Liversedge, S. P., Pollatsek, A., \& Rayner, K. (2009). Encoding multiple words simultaneously in reading is implausible. Trends in Cognitive Sciences, 13, 115-119. http://dx.doi.org/10.1016/j.tics.2008.12.002

Snell, J., \& Grainger, J. (2019a). Readers are parallel processors. Trends in Cognitive Sciences, 23, 537-546. http://dx.doi.org/10.1016/j.tics.2019.04 .006

Snell, J., \& Grainger, J. (2019b). Word position coding in reading is noisy. Psychonomic Bulletin \& Review, 26, 609-615. http://dx.doi.org/10.3758/ s13423-019-01574-0

Snell, J., Meeter, M., \& Grainger, J. (2017). Evidence for simultaneous syntactic processing of multiple words during reading. PLOS ONE, 12, e0173720. http://dx.doi.org/10.1371/journal.pone.0173720

Snell, J., van Leipsig, S., Grainger, J., \& Meeter, M. (2018). OB1-reader: A model of word recognition and eye movements in text reading. Psychological Review, 125, 969-984. http://dx.doi.org/10.1037/rev0000119

van Heuven, W. J., Mandera, P., Keuleers, E., \& Brysbaert, M. (2014). SUBTLEX-U.K.: A new and improved word frequency database for British English. Quarterly Journal of Experimental Psychology, 67, 1176-1190. http://dx.doi.org/10.1080/17470218.2013.850521

Wagenmakers, E. J. (2007). A practical solution to the pervasive problems of p values. Psychonomic Bulletin \& Review, 14, 779-804. http://dx.doi .org/10.3758/BF03194105

Received January 10, 2019

Revision received August 28, 2019 Accepted August 29, 2019 